Received: 13 February 2017

Accepted: 7 April 2017

Published online: 19 May 2017

\section{OPEN A heteromeric molecular complex regulates the migration of lung alveolar epithelial cells during wound healing}

Manik C. Ghosh ${ }^{1}$, Patrudu S. Makena ${ }^{2}$, Joseph Kennedy ${ }^{1}$, Bin Teng ${ }^{1}$, Charlean Luellen ${ }^{1}$, Scott E. Sinclair ${ }^{1,2}$ \& Christopher M. Waters $\mathbb{D}^{1,2}$

Alveolar type II epithelial cells (ATII) are instrumental in early wound healing in response to lung injury, restoring epithelial integrity through spreading and migration. We previously reported in separate studies that focal adhesion kinase-1 (FAK) and the chemokine receptor CXCR4 promote epithelial repair mechanisms. However, potential interactions between these two pathways were not previously considered. In the present study, we found that wounding of rat ATII cells promoted increased association between FAK and CXCR4. In addition, protein phosphatase-5 (PP5) increased its association with this heteromeric complex, while apoptosis signal regulating kinase-1 (ASK1) dissociated from the complex. Cell migration following wounding was decreased when PP5 expression was decreased using shRNA, but migration was increased in ATII cells isolated from ASK1 knockout mice. Interactions between FAK and CXCR4 were increased upon depletion of ASK1 using shRNA in MLE-12 cells, but unaffected when PP5 was depleted. Furthermore, we found that wounded rat ATII cells exhibited decreased ASK1 phosphorylation at Serine-966, decreased serine phosphorylation of FAK, and decreased association of phosphorylated ASK1 with FAK. These changes in phosphorylation were dependent upon expression of PP5. These results demonstrate a unique molecular complex comprising CXCR4, FAK, ASK1, and PP5 in ATII cells during wound healing.

Epithelial repair mechanisms are initiated immediately following lung injury and involve an acute inflammatory response, immune cell recruitment, and activation of the coagulation cascade (reviewed in ref. 1). Nearby facultative progenitor cells, primarily alveolar type II cells (ATII) in the alveolus, rapidly migrate and spread to cover the denuded surface, while circulating stem cells and other progenitor cells are later recruited to the site of injury ${ }^{2-6}$. Along with these recruited cells, ATII cells eventually proliferate and undergo phenotypic differentiation in order to re-establish the integrity and functional organization of the epithelial layer ${ }^{7-12}$. Thus, it is clear that epithelial repair is a dynamic process involving primarily cell spreading and cell migration in the early stages, and later involves recruitment, proliferation, and differentiation.

Focal adhesion kinase-1 (FAK), a non-receptor tyrosine kinase, has long been recognized as a key regulator of cell migration (reviewed in refs 13-15). We and others have previously shown that overexpression of FAK stimulates cell migration, while decreased expression or overexpression of negative regulators inhibits cell migration $^{16-20}$. FAK regulates cell migration in response to a broad range of stimuli and through multiple signaling pathways, most prominently the Src family of kinases. Interactions with integrin receptors increases phosphorylation of FAK at Tyr ${ }^{397}$ which promotes binding of Src and the formation of complexes with other structural and signaling molecules ${ }^{13,21,22}$. For example, we previously found that cell migration in a scratch wound model was dependent upon FAK interactions with c-jun N-terminal kinase (JNK) mediated via JNK-interacting protein-3 $(\mathrm{JIP} 3)^{17}$. Through these complexes, FAK promotes several elements of cell migration including membrane protrusion and focal adhesion turnover.

We recently demonstrated that wounded ATII cells secreted the chemokine CXCL12 which promoted cell migration and wound closure through binding to its receptor CXCR4 ${ }^{23}$. CXCL12/CXCR4-induced cell migration

${ }^{1}$ Department of Physiology, University of Tennessee Health Science Center, Memphis, TN, 38163, USA. ${ }^{2}$ Department of Medicine, University of Tennessee Health Science Center, Memphis, TN, 38163, USA. Correspondence and requests for materials should be addressed to C.M.W. (email: cwaters2@uthsc.edu) 
was previously demonstrated in progenitor B cells to be dependent upon interactions between CXCR4 and FAK ${ }^{24,25}$. However, although it was reported that CXCL12 stimulated the activation of FAK and its recruitment into lipid rafts, the molecular interactions between FAK and CXCR4 were not elucidated. In the current study we investigated the interactions between FAK and CXCR4 in migrating ATII cells following wounding. Also, since our previous studies identified FAK-mediated regulation of JNK in lung epithelial cell migration ${ }^{17}$, we hypothesized that apoptosis signal regulating kinase-1 (ASK1), which activates JNK, is part of the FAK complex that regulates cell migration. Knockdown or inhibition of ASK1 has been shown to either promote ${ }^{26}$ or diminish ${ }^{27,28}$ cell $^{2}$ migration in tumor cells, but this has not previously been investigated in ATII cells. Since these interactions may be dependent upon changes in phosphorylation of ASK1, we also investigated the role of protein phosphatase-5 (PP5), a key regulator of ASK1 activity ${ }^{29,30}$. We identified a molecular complex of FAK, CXCR4, PP5, and ASK1 that changed in composition in cells following wounding and that was dependent upon changes in phosphorylation of both FAK and ASK1.

\section{Results}

FAK interactions are altered in ATII cells following wounding. To determine whether CXCR4 interacts with FAK in ATII cells, we performed immunoprecipitation (IP) studies in unwounded rat ATII cells and in cells $24 \mathrm{hr}$ after multiple scratch wounds were applied to enrich the population of migrating cells. Figure 1A shows that CXCR4 interacted with FAK under control (unwounded) conditions, but the interaction increased in cells following wounding. These results were confirmed by immunoprecipitation with both a FAK antibody and a CXCR4 antibody followed by immunoblotting. Figure 1B provides quantitation for interactions using IP for FAK, indicating a significant increase in FAK/CXCR4 interactions in wounded cells. Similar to CXCR4, the interaction between PP5 and FAK was significantly increased in wounded cells. In contrast, the association between ASK1 and FAK was significantly decreased in wounded cells compared with control cells. These interactions were confirmed using IP with PP5 and ASK1 antibodies followed by immunoblotting. The results demonstrate basal interactions between FAK, CXCR4, ASK1, and PP5 that are enhanced in migrating cells for FAK, CXCR4, and PP5, but are diminished for ASK1.

Wound healing is stimulated in cells deficient in ASK1, but inhibited in cells deficient in PP5. We previously showed that over-expression of FAK promoted epithelial wound closure while expression of a dominant negative form of FAK (FRNK) inhibited wound closure ${ }^{17,18}$. We also showed that cells deficient in expression of CXCR4 exhibited decreased cell migration following wounding ${ }^{23}$. To determine whether decreased expression of ASK1 or PP5 would alter wound closure, we measured wound closure in a scratch wound assay in primary ATII cells from wild type and ASK1-/- mice and in MLE-12 cells expressing either control shRNA or PP5 shRNA. Figure 2 shows that ASK1 was not expressed in ATII cells from ASK1-/- mice and that PP5 expression was decreased in PP5-shRNA-MLE-12 cells (by approximately 40\%, data not shown). Wound closure was significantly accelerated (smaller wounds at $24 \mathrm{hr}$ ) in ASK1-/- ATII cells compared with wild type ATII cells (Fig. 2A). MLE-12 cells deficient in expression of PP5 exhibited decreased wound closure (Fig. 2B).

CXCR4-FAK interactions are inhibited by ASK1. To determine whether the interactions between CXCR4 and FAK were affected by ASK1, IP experiments were performed using MLE-12 cells with shRNA knockdown of ASK1 ( $65 \%$ reduction, data not shown) and with primary ATII cells from ASK1-/- mice. Figure 3 shows that CXCR4 immunoprecipitated with FAK was significantly increased when ASK1 expression was decreased by shRNA (Fig. 3A) or knockout (Fig. 3B). There was no effect on CXCR4/FAK interactions when PP5 expression was decreased by shRNA (Fig. 3A).

Wounding promotes decreased serine phosphorylation of FAK and ASK1. We previously showed an increase in $\mathrm{Tyr}^{397}$ phosphorylation following wounding of lung epithelial cells ${ }^{17}$. However, the role of serine phosphorylation in the regulation of FAK and its interactions with other proteins is less well understood ${ }^{31,32}$. To determine whether serine phosphorylation of FAK was altered in migrating cells, we used a FAK antibody for IP followed by western blotting for phospho-serine ( $\mathrm{p}$-Ser). When we used the monoclonal anti-phospho-serine antibody 1C8, we detected a significant decrease in FAK p-Ser in wounded cells compared with unwounded cells (Fig. 4A).

To determine whether ASK1 serine phosphorylation was altered in migrating rat ATII cells, we used an ASK1 antibody for IP and then immunoblotted using an antibody against $\mathrm{p}$-Ser ${ }^{966}$-ASK1. Wounding caused a significant decrease in p-Ser ${ }^{966}$-ASK1 (Fig. 4B). Using a similar approach, we found no changes in p-Ser ${ }^{83}$-ASK1 following wounding (data not shown). Furthermore, when we used a FAK antibody for IP, there was a significant decrease in p-Ser ${ }^{966}$-ASK1 associated with FAK (Fig. 4C).

Loss of serine phosphorylation of FAK and ASK1 in migrating cells is regulated by PP5. Based upon our findings that there was an increase in PP5 association with FAK and a decrease in association with ASK1 in wounded cells (Fig. 1), we investigated whether decreased expression of PP5 affected serine phosphorylation. Figure 5A shows that p-Ser ${ }^{966}$-ASK1 was significantly decreased in unwounded MLE-12 cells with reduced PP5 expression, and there was no significant change in $\mathrm{p}$-Ser ${ }^{966}$-ASK1 in wounded cells with PP5-shRNA. Although there was no significant difference in p-Ser-FAK in cells with control shRNA and PP5-shRNA, wounding of cells with PP5-shRNA caused a significant increase in p-Ser-FAK (Fig. 5B).

\section{Discussion}

The early stages of wound repair in the lungs involves spreading and migration of epithelial cells to cover the denuded surface. This repair involves complex processes in which focal adhesion complexes alternately form and release attachments with the underlying substrate, and FAK has been shown to be a crucial component of protein 


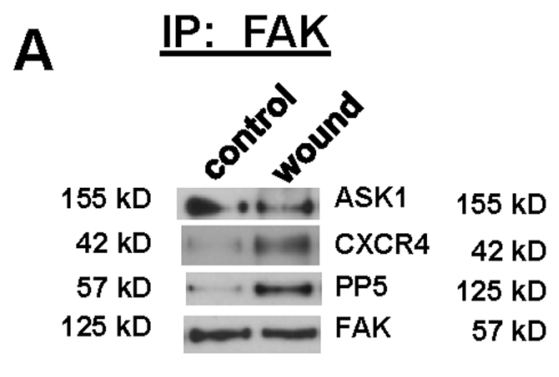

IP: PP5

IP: CXCR4
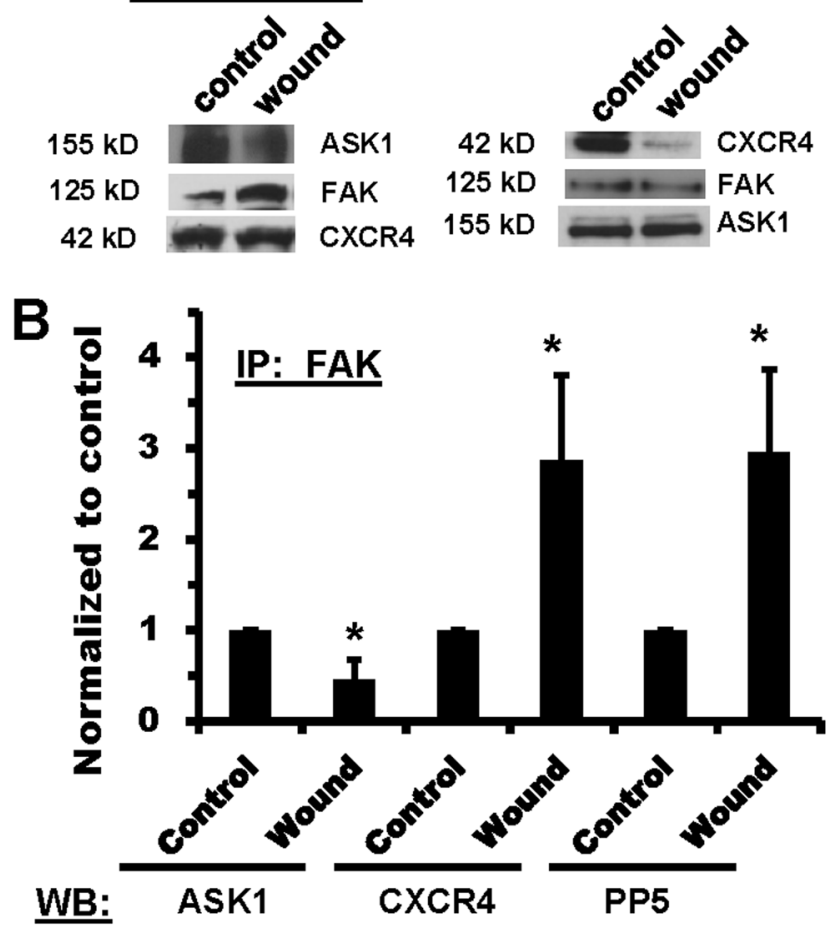

Figure 1. FAK interactions with CXCR4, ASK1, and PP5 were altered in wounded rat ATII cells. Cell lysates were collected from either unwounded monolayers or monolayers $24 \mathrm{hr}$ after multiple scratch wounds to enrich for migrating cells. Equal amounts of protein were immunoprecipitated (IP) from cell lysates using antibodies against FAK, CXCR4, ASK1, and PP5, and then separated by gel electrophoresis followed by immunoblotting for the indicated proteins. Panel A Representative immunoblots showing interactions and the response to wounding. Each pair of bands shown was cropped from the same blot, and the full-length blots were provided in supplemental data. Each protein indicated was immunoblotted separately from independent gels. Panel B Densitometry analysis of results from FAK IP and immunoblot for ASK1, CXCR4, and PP5. Densitometry values were first normalized to FAK and then to the control unwounded condition. Asterisks $(*)$ indicate a significant difference compared to control; $(\mathrm{n}=4), \mathrm{p}<0.05$.

complexes in focal adhesions ${ }^{13,14}$. Using an approach in which we enriched the population of migrating cells in a multiple scratch wound model, we identified for the first time that CXCR4 and PP5 increased their interactions with FAK in migrating cells, while ASK1 dissociated from this complex. In addition, we showed that migrating cells exhibited decreased phosphorylation of ASK1 at Ser-966 and decreased serine phosphorylation of FAK.

FAK mediates cell migration through its involvement in membrane protrusion and turnover of focal adhesions, and the signaling pathways involved in these processes are complex and overlapping. Based upon our previous studies examining lung epithelial repair mechanism $\mathrm{s}^{16-18,23}$ and other studies demonstrating interactions in progenitor B cells ${ }^{24,25}$, we hypothesized that CXCR4 signaling interacted with FAK during cell migration, and our results demonstrated an increase in association between CXCR4 and FAK in a population of cells enriched in migrating cells (Fig. 1). CXCR4 is a ubiquitous chemokine receptor that is involved in the activation of actin polymerization and initiation of cell migration in different types of cells, including ATII cells ${ }^{33-35}$. We previously showed that both inhibition and decreased expression of CXCR4 led to significant inhibition of cell migration in scratch wound models ${ }^{23}$. The prior studies in B cells showed that CXCL12 promoted FAK translocation into lipid rafts ${ }^{24,25}$, but these studies did not demonstrate the molecular interaction between FAK and CXCR4, either by co-localization or by immunoprecipitation. Because CXCR4 is a G-protein coupled receptor with seven 

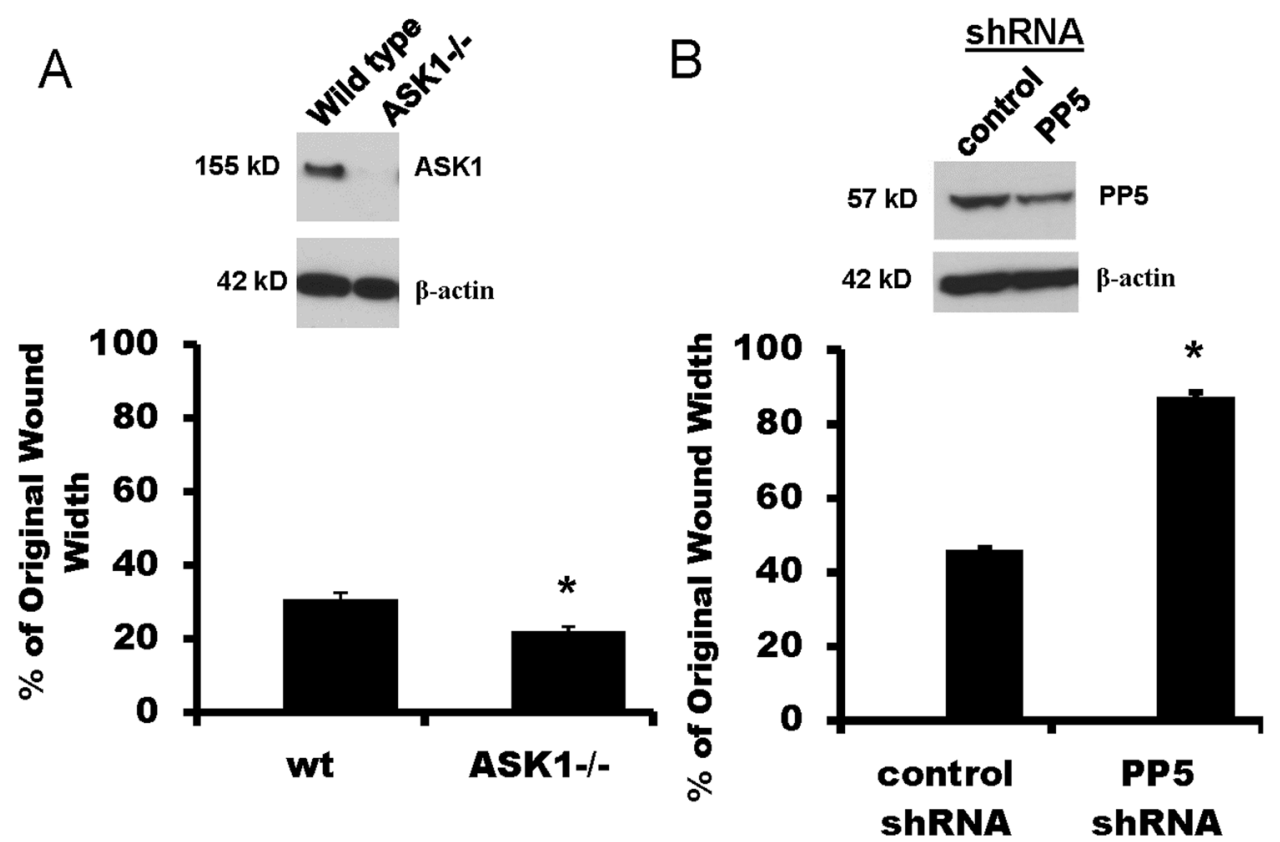

Figure 2. Wound healing was stimulated in ASK1-deficient cells, but inhibited in PP5-deficient cells. Scratch wounds were applied to confluent monolayers, and the wound widths were measured $24 \mathrm{hr}$ later as a percentage of the original wound width. Panel A Primary ATII cells from ASK1-/ - mice exhibited accelerated wound closure compared with ATII cells from wild type (wt) mice; wound closure measurements were made using the IncuCyte ZOOM system. Expression of ASK1 relative to $\beta$-actin is shown in the upper panel. Panel B Wound closure was inhibited in MLE-12 cells with PP5 expression knocked down by shRNA compared with control shRNA cells; wound closure was measured following multiple pipette tip scratch wounding. Expression of PP5 (relative to $\beta$-actin) was reduced by $40 \%$ (upper panel). Asterisks $(*)$ indicate a significant difference compared to control; ( $\mathrm{n}=14$ for Ask $1-/-$ and $\mathrm{n}=6$ for PP5 shRNA), $\mathrm{p}<0.05$. Each pair of bands shown was cropped from the same blot, and the full-length blots were provided in supplemental data.

transmembrane domain regions, the increased interactions between FAK and CXCR4 most likely occurs at the plasma membrane, but we did not determine whether this occurs in lipid rafts or other regions.

In addition to the interaction between FAK and CXCR4, our immunoprecipitation studies showed basal interactions between FAK, CXCR4, PP5, and ASK1 in quiescent (unwounded) cells, but increased interactions among FAK, CXCR4, and PP5, and decreased interactions with ASK1, in migrating cells following wounding (Fig. 1). These results suggest increased assembly of a complex containing FAK, CXCR4, and PP5 that promotes cell migration and repair, with a corresponding loss of ASK1 from the complex. In support of these findings, and to demonstrate functional relevance, knockdown of PP5 using shRNA significantly inhibited cell migration, while ATII cells from ASK1-/- mice exhibited accelerated wound closure (Fig. 2). Furthermore, knockdown of ASK1 by shRNA or deficiency in ASK1-/- cells significantly increased the association between FAK and CXCR4 in unwounded cells (Fig. 3). In contrast, knockdown of PP5 had no effect on FAK-CXCR4 interactions (Fig. 3A). While the formation of complexes with FAK, integrins, and other signaling molecules in migrating cells has been extensively studied, there are no previous reports of interactions between FAK, CXCR4, PP5, and ASK1 regulating cell migration.

ASK1 is ubiquitously expressed and is known to regulate p38- and JNK-mediated inflammation and apop$\operatorname{tosis}^{36,37}$. Because our previous studies demonstrated FAK-dependent activation of JNK in migrating epithelial cells $^{18}$, we hypothesized that ASK1 was involved in the regulation of JNK-mediated motility. In our studies with lung epithelial cells, deficiency of ASK1 stimulated cell migration, with a corresponding increase in interactions between FAK and CXCR4 (Figs 2 and 3). Inhibition of ASK1 was previously shown to inhibit cell motility in both oral squamous carcinoma cells ${ }^{27}$ and chondrosarcoma cells ${ }^{28}$, but knockdown of ASK1 in breast cancer cells stimulated cell migration ${ }^{26}$. Thus, the regulatory role of ASK1 in cell migration appears to vary among cells from different tissues, similar to its tissue-specific regulation of inflammation and apoptosis. A limitation of our approach is that we have interpreted wound closure after $24 \mathrm{hr}$ as an index of primarily cell spreading and migration, but cell proliferation can also contribute to wound closure over longer periods of time. Our previous measurements with primary rat ATII cells showed that only $\sim 13 \%$ of cells near the wound edge had incorporated ethidium deoxyuridine (as an index of proliferation) by $24 \mathrm{hr}^{38}$, suggesting a relatively small contribution of proliferation to wound closure at that time.

ASK1 interacts with a significant number of intracellular proteins with an extensive range of functions, and the term ASK1 signalosome has been used to describe a high molecular mass complex $(>1500 \mathrm{kDa})$ that forms in cells ${ }^{39,40}$. ASK1 also contains multiple phosphorylation sites that regulate function, activity, and association with other proteins ${ }^{39,41}$. Activation of ASK1 is reported to be regulated by phosphorylation of a threonine residue ( $\mathrm{Thr}^{838}$ for human, $\mathrm{Thr}^{845}$ for mice) within the kinase domain. In addition phosphorylation of serine residues 

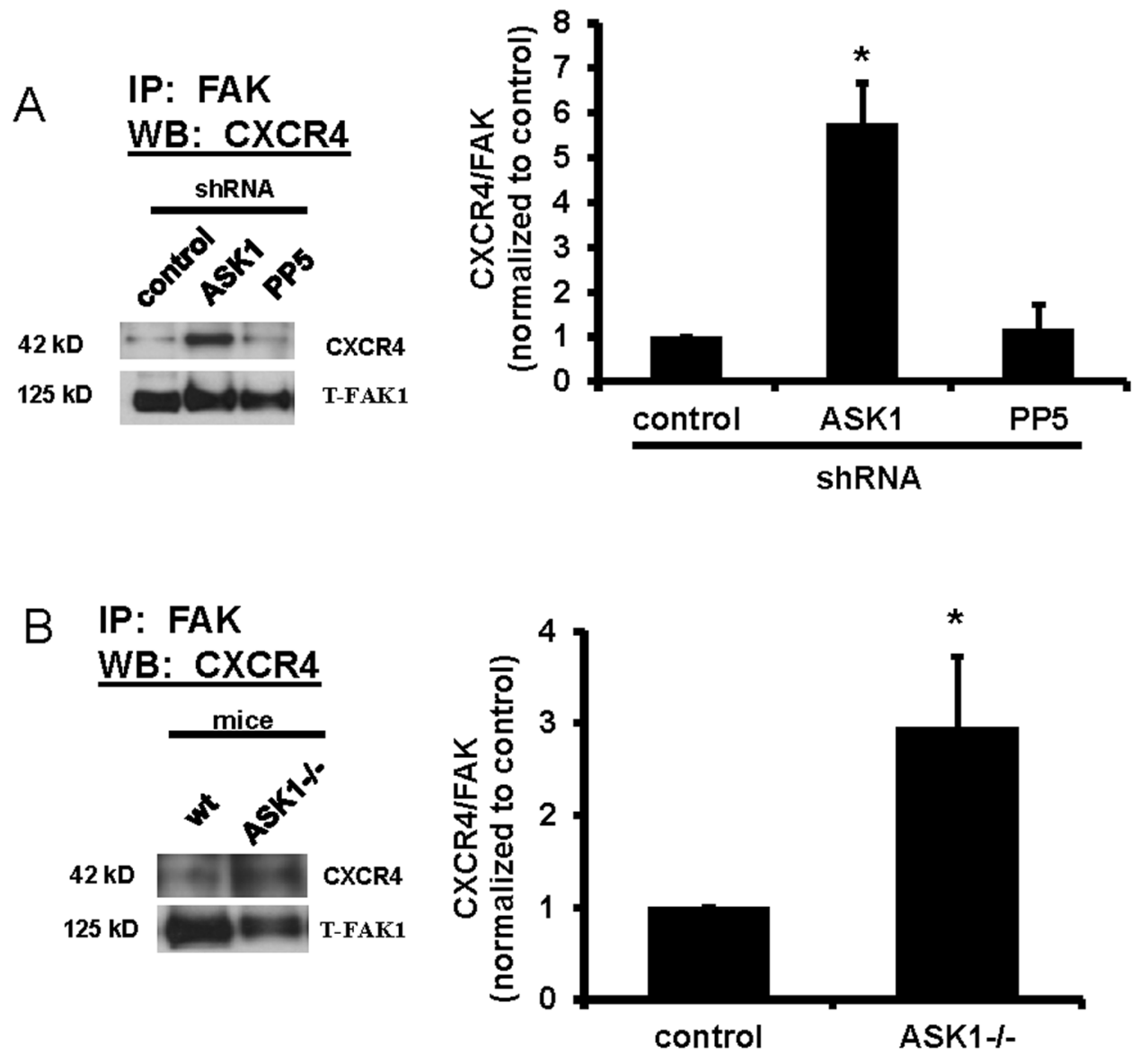

Figure 3. CXCR4 interactions with FAK were inhibited by ASK1. Panel A Cell lysates were collected from MLE-12 cells with control shRNA, ASK1-shRNA, or PP5-shRNA, immunoprecipitated with a FAK antibody, and immunoblotted for CXCR4 or FAK. Decreased expression of ASK1 caused a significant increase in CXCR4 associated with FAK as indicated by densitometry analysis (right panel), but decreased expression of PP5 did not affect the interaction $(\mathrm{n}=3)$. Panel B CXCR4 interaction with FAK was significantly increased in cell lysates from ATII cells from ASK1-/- mice compared with cells from wild type (wt) mice $(\mathrm{n}=5)$. Asterisks (*) indicate a significant difference compared to control (control-shRNA or wild type); $\mathrm{p}<0.05$. Total FAK was immunoblotted as a loading control. Each set of bands shown was cropped from the same blot, and the fulllength blots were provided in supplemental data.

$\mathrm{Ser}^{83}, \mathrm{Ser}^{966}$, and $\operatorname{Ser}^{1033}$ cause association of regulatory molecules that result in inactivation of ASK1. We found that wounding caused a significant decrease in the content of p-Ser ${ }^{966}$-ASK1 in cells (Fig. 4B), and this corresponded with a significant decrease in p-Ser ${ }^{966}$-ASK1 associated with FAK (Fig. 4C). In contrast the content of p-Ser ${ }^{83}$-ASK1 was unaffected by wounding (data not shown). Due to the limitations of available antibodies, we did not determine whether $\mathrm{p}-\mathrm{Ser}^{1033}$-ASK1 was affected by wounding. These results suggest that dephosphorylation of ASK1 at Ser ${ }^{966}$ in wounded cells promotes dissociation of ASK1 from the FAK/CXCR4/PP5 complex. The change in phosphorylation may lead to changes in conformation and activation of ASK1. For example, phosphorylation of Ser ${ }^{966}$ has been shown to facilitate the association of ASK1 with the 14-3-3 family of proteins and subsequent decreased ASK1 activity ${ }^{42-44}$. We did not determine whether 14-3-3 proteins were part of the FAK/ CXCR4/PP5/ASK1 complex. The purpose of ASK1 dissociation and the mechanisms by which this promotes cell migration remain to be determined.

In addition to the changes in ASK1 phosphorylation following wounding, we also detected a decrease in p-Ser on FAK using the 1C8 antibody. FAK contains 25 sites for phosphorylation, including 15 serine, 5 threonine, and 5 tyrosine residues ${ }^{32}$. Conformation changes in FAK following tyrosine phosphorylation at different sites has been shown to regulate integrin engagement and interactions with other signaling molecules, notably the creation of high affinity binding sites following phosphorylation at $\operatorname{Tyr}^{397}$ (reviewed in refs 15, 45-47). As stated above there are few studies on the role of serine phosphorylation of $\mathrm{FAK}^{31,32}$. We used a phosphoserine detection kit to investigate changes in serine phosphorylation on FAK following wounding. Of the panel of antibodies in the kit, only the 1C8 monoclonal antibody indicated a difference in p-Ser-FAK following wounding (Fig. 4A). However, we do not know at this time which of the 15 serine sites on FAK exhibited this change in phosphorylation.

Because we observed loss of serine phosphorylation on both FAK and ASK1 we investigated whether PP5 regulated these events. PP5 is a member of the serine/threonine phosphatase family of proteins, and has been shown 

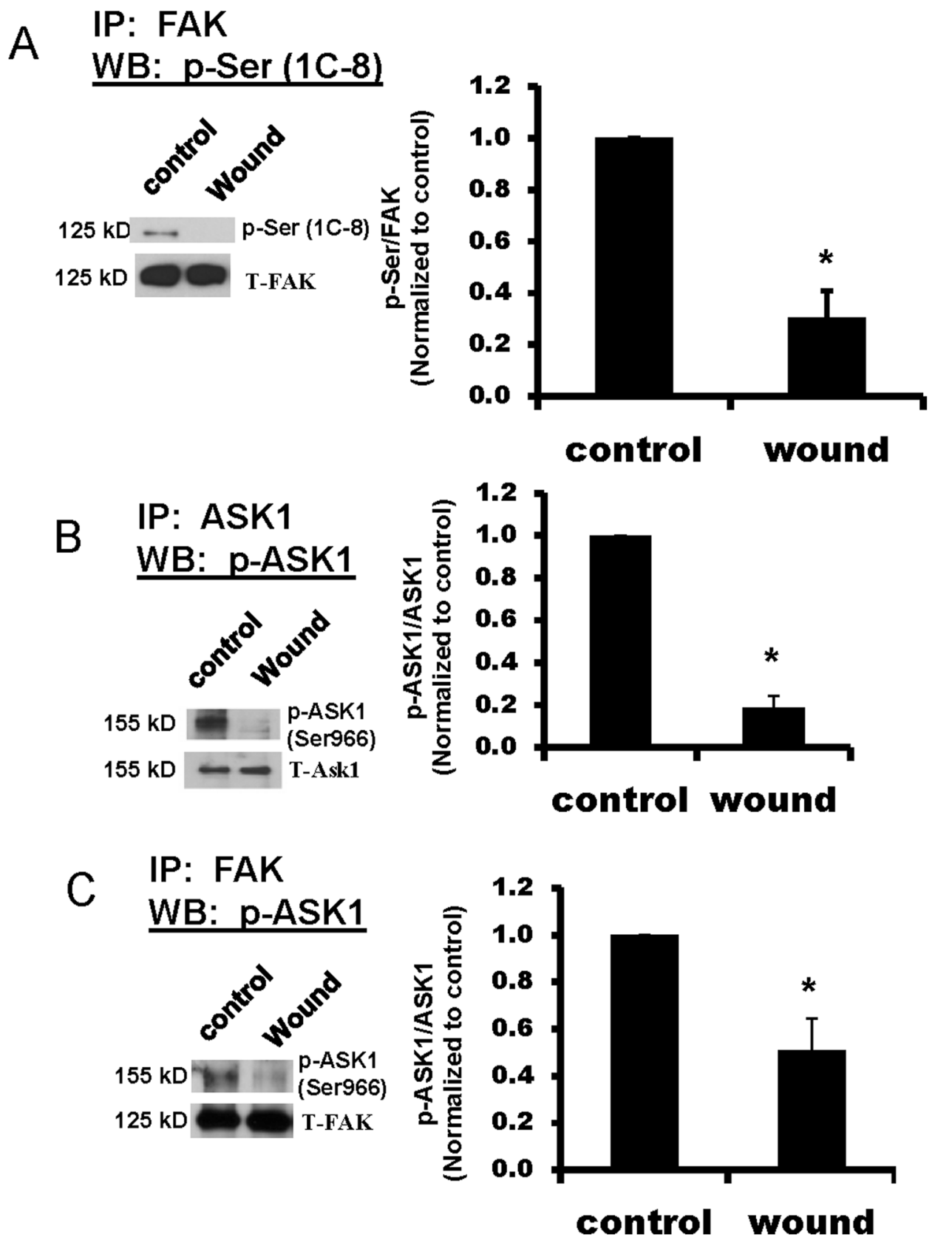

Figure 4. Serine phosphorylation of FAK and ASK1 were decreased in wounded cells. Cell lysates from unwounded and wounded primary rat ATII cells were collected after $24 \mathrm{hr}$, immunoprecipitated (IP) for FAK or ASK1, and then immunoblotted as indicated. Panel A Wounding caused a significant decrease in p-Ser-FAK recognized using the $1 \mathrm{C} 8$ antibody. Panel B Wounding caused a significant decrease in p-Ser ${ }^{966}$-ASK1. Panel C Wounding caused a significant decrease in p-Ser ${ }^{966}$-ASK1 associated with FAK. Left panels show representative blots, and right panels show densitometry with values normalized first to the loading control and then to the control unwounded condition. Asterisks $(*)$ indicate a significant difference compared to control; $(\mathrm{n}=4)$, $\mathrm{p}<0.05$. Each pair of bands shown was cropped from the same blot, and the full-length blots were provided in supplemental data. Each protein indicated was immunoblotted separately from independent gels.

to be involved in numerous cellular processes, including MAPK-mediated signaling ${ }^{48,49}$. PP5 has been reported to interact with ASK1 and cause dephosphorylation at Thr- $845^{29,50}$. We found that shRNA-induced knockdown of PP5 caused decreased p-Ser ${ }^{966}$-ASK1 in basal cells (Fig. 5A). In contrast with control cells, wounding did not cause a decrease in $\mathrm{p}$-Ser ${ }^{966}$-ASK1 in cells deficient in PP5. Thus, PP5 may regulate the baseline association of ASK1 within the complex, but it does not appear to regulate its dissociation following wounding of cells. There is some evidence that PP2B may also regulate phosphorylation of $\mathrm{ASK} 1^{51}$, but we did not investigate PP2B in this study. Wounding also caused a decrease in p-Ser-FAK phosphorylation in rat ATII cells (Fig. 4A). While we did not observe a significant decrease in p-Ser-FAK in control MLE-12 cells following wounding (Fig. 5B), we did 
A

\section{IP: ASK1}

WB: p-ASK1

$155 \mathrm{kD}$

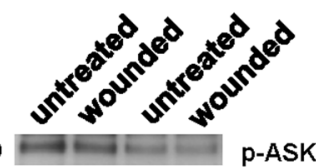

$155 \mathrm{kD}$

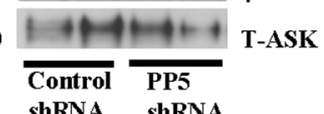

B

IP: FAK

WB: $\mathrm{p}-\operatorname{Ser}(1 \mathrm{C}-8)$

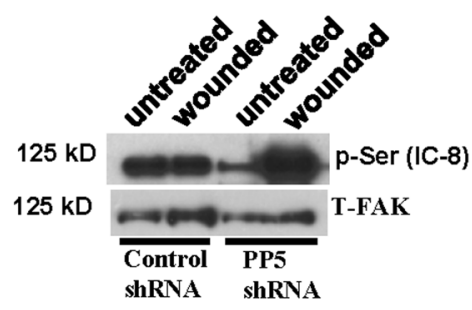

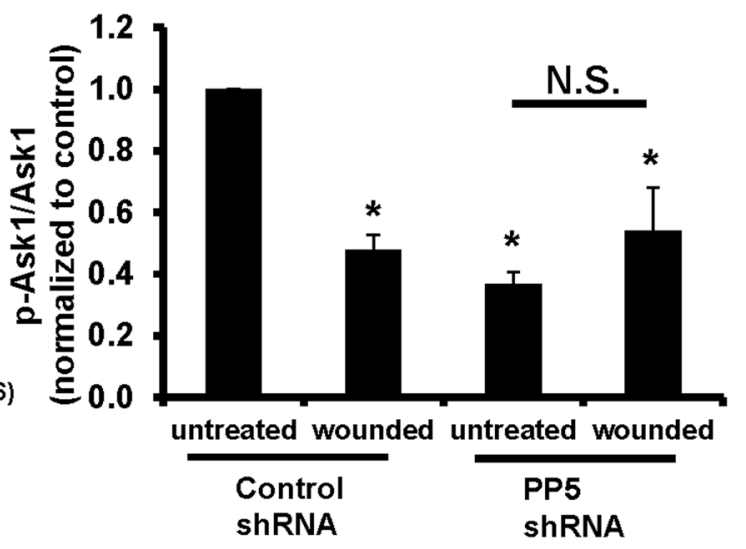

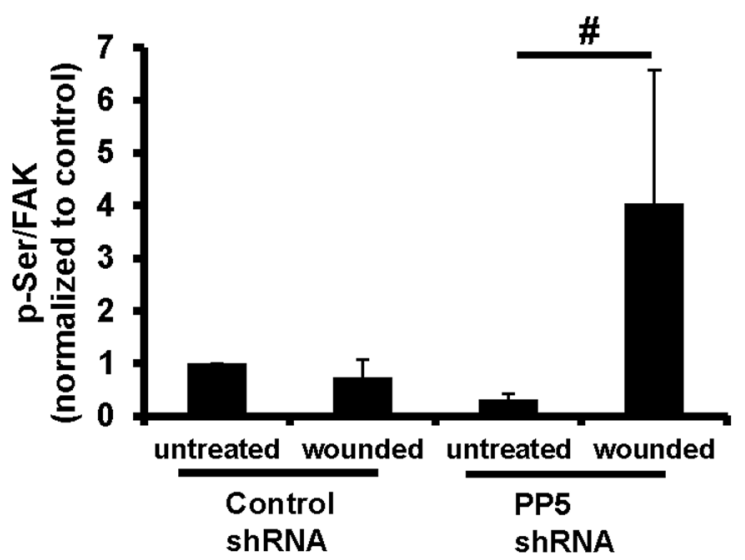

Figure 5. PP5 regulates the phosphorylation of FAK and ASK1 in wounded cells. Cell lysates from unwounded and wounded MLE-12 cells were collected after $24 \mathrm{hr}$, immunoprecipitated (IP) for FAK or ASK1, and then immunoblotted as indicated. Panel A Knockdown of PP5 using shRNA decreased p-Ser ${ }^{966}$-ASK1 in unwounded cells and showed no change in wounded cells. Panel B Knockdown of PP5 using shRNA caused a significant increase in p-Ser-FAK (recognized by the $1 \mathrm{C} 8$ antibody) in wounded cells. Right panels show the densitometry analysis normalized to unwounded controls. Asterisks (*) indicate a significant difference compared to control, $(\mathrm{n}=4), \mathrm{p}<0.05$. N.S. - no significant difference. Each set of bands shown was cropped from the same blot, and the full-length blots were provided in supplemental data. Each protein indicated was immunoblotted separately from independent gels.

see a significant increase in p-Ser-FAK following wounding of MLE-12 cells with PP5-shRNA knockdown. These results suggest that PP5 regulates the phosphorylation of FAK at the serine site recognized by the $1 \mathrm{C} 8$ antibody. Further analysis using antibodies with greater specificity and mass spectrometry will be necessary to specifically identify which site on FAK is regulated and whether there are other phosphatases involved.

The changes in serine phosphorylation on ASK1 and FAK, and the regulation by PP5, point toward an important role for serine kinases in the regulation of cell migration. It is well-established that phosphoinositide- 3 kinase (PI-3 kinase) and Akt promote cell migration through serine phosphorylation, including in bronchial epithelial cells $^{52,53}$. Earlier studies also demonstrated that activation of protein kinase A accelerates bronchial epithelial cell wound closure ${ }^{54}$, while activation of protein kinase $\mathrm{C}$ inhibits wound closure ${ }^{55}$, ${ }^{6}$. While these two serine/ threonine kinases may undergo bi-directional regulation, these processes may also be regulated in part by protein phosphatases. We did not investigate the regulation of these kinases in the context of the molecular complex described above.

In summary, our results reveal novel interactions among FAK, CXCR4, ASK1, and PP5 that regulate cell migration following wounding. Under quiescent conditions, these molecules are associated with one another, as well as other proteins that may be part of the complex. Following injury to the epithelial cells, there is greater association between FAK, CXCR4, and PP5, but ASK1 dissociates from the complex in migrating cells. The dissociation of ASK1 corresponds with a decrease in phosphorylation of ASK1 at Ser ${ }^{83}$ and a decrease in serine phosphorylation of FAK at a site recognized by the 1C8 antibody. These molecular interactions may be important in the repair of the epithelium following injury to the lungs due to their role in efficient cell migration. In this context, acute or repetitive injuries that disrupt these signaling pathways and molecular interactions could result in dysfunctional repair, and conversely, promotion of these pathways and interactions could promote repair. 


\begin{abstract}
Methods
Reagents. Dulbecco's Modified Eagle's Medium (DMEM), penicillin-streptomycin, trypsin-ethylenediaminetetraacetic acid (EDTA) solution, and phosphate buffer saline were purchased from GIBCO Life Technologies (Grand Island, NY). Fetal bovine serum (FBS) was obtained from Hyclone (Logan, UT). 4-(2-hydroxyethyl)-1-piperazineethanesulfonic acid (HEPES) was purchased from Gibco (Grand Island, NY). Tween-20 was purchased from Bio-Rad (Hercules, CA). Gradient gels (4-12\%) for Western blot were purchased from Invitrogen Inc. (Carlsbad, CA). Antibodies against CXCR4 were purchased from abCAM (Cambridge, MA, cat. \#ab13854). Antibodies against focal adhesion kinase-1 (FAK) were purchased from BD-Bioscience (San Jose, CA). Antibodies against protein phosphatase-5 (PP5) were purchased from Santa Cruz Biotech (Santa Cruz, CA). Antibodies against apoptosis signal regulating kinase-1 (ASK1) were purchased from Genetex Inc. (Irvine, CA). A monoclonal antibody against phophoserine (clone 1C8) was purchased as part of a phosphoserine detection kit from EMD Millipore (San Diego, CA, cat. \#525282).
\end{abstract}

Cell culture. Primary rat ATII cells were isolated according to the methods described previously ${ }^{57,58}$. The animal use protocol was approved by the Institutional Animal Care and Use Committee at the University of Tennessee Health Science Center. Briefly, ATII cells were isolated from male Sprague-Dawley rats by elastase digestion and differential adherence on IgG-coated dishes. ATII cells were identified using Nile Red (Sigma) staining of lamellar bodies, and $>95 \%$ of the cells were Nile Red-positive on day 2 . We used plastic 6-well plates or chamber slides that were coated with rat lung fibroblast (RFL) matrix deposited by RLF- 6 cells (American Type Culture Collection) for wound healing assays with ATII cells ${ }^{57}$. Freshly isolated cells were seeded to confluence at $3.5 \times 10^{6}$ cells/well in ATII culture medium (DMEM with $10 \% \mathrm{FBS}, 4 \mathrm{mM}$ glutamine, $1 \%$ penicillin/streptomycin, and $0.25 \mu \mathrm{M}$ amphotericin B), and experiments were performed on day 2 after isolation. MLE- 12 cells, a transformed cell line of mouse alveolar epithelial cells ${ }^{59}$, were cultured using media containing DMEM with $10 \%$ FBS, $4 \mathrm{mM}$ glutamine, $1 \%$ penicillin/streptomycin.

Isolation of AT2 cell from wild type and ASK1-Knockout mice. ATII cells from wild type and ASK1 knockout mice (ASK1-/-) were isolated according to previous methods ${ }^{60}$. The yield per mouse lung was 3 to 5 million with $>90 \%$ purity assessed by immuno-staining for surfactant protein-C. The animal use protocol for these studies was approved by the Institutional Animal Care and Use Committee at the University of Tennessee Health Science Center (UTHSC). All experiments were performed in accordance with UTHSC guidelines and regulations.

Development of ASK1 and PP5-shRNA cell lines. Stable cell lines with decreased expression of ASK1 and PP5 were developed in A549 (ASK1) and MLE-12 (PP5) cells using shRNA (SantaCruz Biotech, ASK1-Cat\# sc-29749-V and PP5-Cat\# sc-44603-V) according to the manufacturer's protocol. A cell line with control shRNA was developed using a scrambled vector (SantaCruz Biotech, Cat\# SC-108080). The antibiotic puromycin was used in the media to select the positive clone. The knockdown status was assessed by western blotting for protein expression.

Wound healing assay. Cell migration was measured according to our previous methods ${ }^{61}$. Confluent monolayers of ATII, A549, MLE12, or shRNA knockdown cells were wounded by scraping multiple pipette tips across the monolayer to produce initial wounds of 1000-1,200 $\mu \mathrm{m}$. Images were collected with a Cool Snap charge-coupled device camera (Roper Scientific, Trenton, NJ) mounted on an Eclipse TE300 inverted microscope with a $4 \mathrm{X}$ objective (Nikon, Melville, NY). Images were obtained at the initial time of wounding and then $24 \mathrm{hrs}$ post-wounding. Metamorph software (Universal Imaging, Westchester, PA) was used to record the coordinates for each wound location using a computer-controlled stage so that the same location was used for post-wounding measurement, and data were analyzed by Metamorph imaging software. The mean wound width at $24 \mathrm{hrs}$ was calculated and normalized to the original wound width as a percentage of the original width. In some studies we used the IncuCyte ZOOM imaging system (Essen Bioscience, Ann Arbor, MI) to measure wound closure. Scratch wounds were created in all 96 wells of a plate using the Essen Bioscience Woundmaker, and images were captured every 120 minutes and analyzed using Metamorph as described above. All scratch wound results reported are from at least three independent wells from at least two separate experiments $(n=6)$.

Immunoprecipitation and immunoblotting. Confluent monolayers of cells were wounded using a multi-pronged comb so as to generate a large number of wound edges for each well, and after $24 \mathrm{hr}$ of cell migration the cells were lysed using RIPA buffer. Unwounded monolayers were used as controls. The cell lysates were then subjected to immunoprecipitation using antibodies against FAK, CXCR4, PP5, and ASK1. Protein concentration was determined by the Bradford method $^{62}$. Equal amounts of protein were resolved by $4-12 \%$ SDS-PAGE, and electrophoretically transferred onto PVDF membranes. Membranes were blocked for $1 \mathrm{~h}$ in $5 \%$ nonfat milk (Bio-Rad, Hercules, CA) in Tris buffered saline (TBS) containing $0.01 \%$ Tween 20 (TBST) and incubated overnight at $4{ }^{\circ} \mathrm{C}$ with the appropriate primary antibody. Membranes were washed with TBST and then incubated with the secondary antibody for $1 \mathrm{~h}$ at room temperature. Blots were developed on X-ray film using the enhanced chemiluminescence method (ECL) (Amarsham Biosciences, Piscataway, NJ). Reciprocal IP and immunoblot experiments were performed 2 to 4 times for each result shown.

Densitometry of immunoblot. Densitometry of bands obtained by immunoblot was performed using the software Image-J developed by the National Institutes of Health (NIH). Each band was normalized against the corresponding beta-actin, GAPDH, or total expression. These values were then normalized against the control condition. 
Statistical analysis. All values are presented as means \pm standard error (SE). A $t$-test was used when only two groups were compared, and one-way ANOVA with the Holm-Sidak method was performed for comparisons of multiple treatments to determine significant differences between individual conditions. Significant differences were determined based on a threshold of $\mathrm{p}<0.05$. Statistical comparisons were made using Sigmastat 3.5 (Jandel Scientific, San Rafael, CA).

\section{References}

1. Crosby, L. M. \& Waters, C. M. Epithelial repair mechanisms in the lung. American journal of physiology. Lung cellular and molecular physiology 298, L715-731, doi: ajplung.00361.2009 (2010).

2. Krause, D. S. Bone marrow-derived cells and stem cells in lung repair. Proceedings of the American Thoracic Society 5, 323-327, doi:10.1513/pats.200712-169DR (2008).

3. Xian, W. \& McKeon, F. Adult stem cells underlying lung regeneration. Cell Cycle 11, 887-894, doi:10.4161/cc.11.5.19328 (2012).

4. Barkauskas, C. E. et al. Type 2 alveolar cells are stem cells in adult lung. The Journal of clinical investigation 123, 3025-3036, doi:10.1172/JCI68782 (2013).

5. Vaughan, A. E. et al. Lineage-negative progenitors mobilize to regenerate lung epithelium after major injury. Nature 517, 621-625, doi:10.1038/nature14112(2015).

6. Zuo, W. et al. p63(+)Krt5(+) distal airway stem cells are essential for lung regeneration. Nature 517, 616-620, doi:10.1038/ nature13903 (2015).

7. Puchelle, E., Zahm, J. M., Tournier, J. M. \& Coraux, C. Airway epithelial repair, regeneration, and remodeling after injury in chronic obstructive pulmonary disease. Proceedings of the American Thoracic Society 3, 726-733 (2006).

8. Stripp, B. R. \& Reynolds, S. D. Maintenance and repair of the bronchiolar epithelium. Proceedings of the American Thoracic Society 5,328-333, doi:10.1513/pats.200711-167DR (2008).

9. Ware, L. B. \& Matthay, M. A. The acute respiratory distress syndrome. The New England journal of medicine 342, 1334-1349 (2000).

10. Zahm, J. M., Chevillard, M. \& Puchelle, E. Wound repair of human surface respiratory epithelium. American Journal of Respiratory Cell \& Molecular Biology 5, 242-248 (1991).

11. Zahm, J. M. et al. Cell migration and proliferation during the in vitro wound repair of the respiratory epithelium. Cell Motility \& the Cytoskeleton 37, 33-43 (1997).

12. Zahm, J. M., Pierrot, D., Chevillard, M. \& Puchelle, E. Dynamics of cell movement during the wound repair of human surface respiratory epithelium. Biorheology 29, 459-465 (1992).

13. Schaller, M. D. Cellular functions of FAK kinases: insight into molecular mechanisms and novel functions. Journal of cell science 123, 1007-1013, doi:10.1242/jcs.045112 (2010)

14. Wehrle-Haller, B. Assembly and disassembly of cell matrix adhesions. Current opinion in cell biology 24, 569-581, doi:10.1016/j. ceb.2012.06.010 (2012).

15. Zhao, X. \& Guan, J. L. Focal adhesion kinase and its signaling pathways in cell migration and angiogenesis. Advanced drug delivery reviews 63, 610-615, doi:10.1016/j.addr.2010.11.001 (2011)

16. Desai, L. P., Sinclair, S. E., Chapman, K. E., Hassid, A. \& Waters, C. M. High tidal volume mechanical ventilation with hyperoxia alters alveolar type II cell adhesion. American journal of physiology. Lung cellular and molecular physiology 293, L769-778 (2007).

17. Desai, L. P., White, S. R. \& Waters, C. M. Mechanical stretch decreases FAK phosphorylation and reduces cell migration through loss of JIP3-induced JNK phosphorylation in airway epithelial cells. American journal of physiology. Lung cellular and molecular physiology 297, L520-529 (2009).

18. Desai, L. P., White, S. R. \& Waters, C. M. Cyclic mechanical stretch decreases cell migration by inhibiting phosphatidylinositol 3-kinase- and focal adhesion kinase-mediated JNK1 activation. The Journal of biological chemistry 285, 4511-4519, doi:10.1074/jbc. M109.084335 (2010).

19. Kodama, K. et al. Laminin 5 expression protects against anoikis at aerogenous spread and lepidic growth of human lung adenocarcinoma. International journal of cancer 116, 876-884, doi:10.1002/ijc.21136 (2005)

20. Mitra, S. K., Hanson, D. A. \& Schlaepfer, D. D. Focal adhesion kinase: in command and control of cell motility. Nature reviews. Molecular cell biology 6, 56-68, doi:10.1038/nrm1549 (2005).

21. Cox, B. D., Natarajan, M., Stettner, M. R. \& Gladson, C. L. New concepts regarding focal adhesion kinase promotion of cell migration and proliferation. Journal of cellular biochemistry 99, 35-52, doi:10.1002/jcb.20956 (2006).

22. Hanks, S. K., Ryzhova, L., Shin, N. Y. \& Brabek, J. Focal adhesion kinase signaling activities and their implications in the control of cell survival and motility. Frontiers in bioscience: a journal and virtual library 8, d982-996 (2003).

23. Ghosh, M. C., Makena, P. S., Gorantla, V. K., Sinclair, S. E. \& Waters, C. M. CXCR4 Regulates Migration of Lung Alveolar Epithelial Cells through Activation of Rac1 and Matrix Metalloproteinase-2 (MMP-2). American journal of physiology. Lung cellular and molecular physiology. doi:10.1152/ajplung.00321.2011 (2012).

24. Le, Y., Honczarenko, M., Glodek, A. M., Ho, D. K. \& Silberstein, L. E. CXC chemokine ligand 12-induced focal adhesion kinase activation and segregation into membrane domains is modulated by regulator of G protein signaling 1 in pro-B cells. J Immunol 174, 2582-2590 (2005).

25. Park, S. Y. et al. Focal adhesion kinase regulates the localization and retention of pro-B cells in bone marrow microenvironments. $J$ Immunol 190, 1094-1102, doi:10.4049/jimmunol.1202639 (2013).

26. Noh, K. T., Cho, S. G. \& Choi, E. J. Knockdown of apoptosis signal-regulating kinase 1 modulates basal glycogen synthase kinase3beta kinase activity and regulates cell migration. FEBS letters 584, 4097-4101, doi:10.1016/j.febslet.2010.08.029 (2010).

27. Chuang, J. Y., Chang, A. C., Chiang, I. P., Tsai, M. H. \& Tang, C. H. Apoptosis signal-regulating kinase 1 is involved in WISP-1promoted cell motility in human oral squamous cell carcinoma cells. PloS one 8, e78022, doi:10.1371/journal.pone.0078022 (2013).

28. Lin, C. Y., Chang, S. L., Fong, Y. C., Hsu, C. J. \& Tang, C. H. Apoptosis signal-regulating kinase 1 is involved in brain-derived neurotrophic factor (BDNF)-enhanced cell motility and matrix metalloproteinase 1 expression in human chondrosarcoma cells. International journal of molecular sciences 14, 15459-15478, doi:10.3390/ijms140815459 (2013).

29. Morita, K. et al. Negative feedback regulation of ASK1 by protein phosphatase 5 (PP5) in response to oxidative stress. The EMBO journal 20, 6028-6036, doi:10.1093/emboj/20.21.6028 (2001)

30. Zhou, G., Golden, T., Aragon, I. V. \& Honkanen, R. E. Ser/Thr protein phosphatase 5 inactivates hypoxia-induced activation of an apoptosis signal-regulating kinase 1/MKK-4/JNK signaling cascade. The Journal of biological chemistry 279, 46595-46605, doi:10.1074/jbc.M408320200 (2004)

31. Chu, M. et al. Serine-910 phosphorylation of focal adhesion kinase is critical for sarcomere reorganization in cardiomyocyte hypertrophy. Cardiovascular research 92, 409-419, doi:10.1093/cvr/cvr247 (2011).

32. Grigera, P. R. et al. FAK phosphorylation sites mapped by mass spectrometry. Journal of cell science 118, 4931-4935, doi:10.1242/ jcs.02696 (2005).

33. Ghosh, M. C., Makena, P. S., Gorantla, V., Sinclair, S. E. \& Waters, C. M. CXCR4 regulates migration of lung alveolar epithelial cells through activation of Racl and matrix metalloproteinase-2. Am J Physiol Lung Cell Mol Physiol 302, L846-856, doi:10.1152/ ajplung.00321.2011 (2012).

34. Guyon, A. CXCL12 chemokine and its receptors as major players in the interactions between immune and nervous systems. Front Cell Neurosci 8, 65, doi:10.3389/fncel.2014.00065 (2014). 
35. Weidt, C. et al. Stem cell migration: a quintessential stepping stone to successful therapy. Curr Stem Cell Res Ther 2, 89-103 (2007).

36. Jibiki, I. et al. Apoptosis signal-regulating kinase 1-mediated signaling pathway regulates nitric oxide-induced activator protein-1 activation in human bronchial epithelial cells. American journal of respiratory and critical care medicine 167, 856-861, doi:10.1164/ rccm.2204042 (2003)

37. Tobiume, K. et al. ASK1 is required for sustained activations of JNK/p38 MAP kinases and apoptosis. EMBO reports 2, 222-228, doi:10.1093/embo-reports/kve046 (2001).

38. Crosby, L. M. et al. Balance of life and death in alveolar epithelial type II cells: proliferation, apoptosis, and the effects of cyclic stretch on wound healing. American journal of physiology. Lung cellular and molecular physiology 301, L536-546, doi:10.1152/ ajplung.00371.2010 (2011).

39. Takeda, K., Noguchi, T., Naguro, I. \& Ichijo, H. Apoptosis signal-regulating kinase 1 in stress and immune response. Annual review of pharmacology and toxicology 48, 199-225, doi:10.1146/annurev.pharmtox.48.113006.094606 (2008).

40. Noguchi, T. et al. Recruitment of tumor necrosis factor receptor-associated factor family proteins to apoptosis signal-regulating kinase 1 signalosome is essential for oxidative stress-induced cell death. The Journal of biological chemistry 280, 37033-37040, doi:10.1074/jbc.M506771200 (2005).

41. Tobiume, K., Saitoh, M. \& Ichijo, H. Activation of apoptosis signal-regulating kinase 1 by the stress-induced activating phosphorylation of pre-formed oligomer. Journal of cellular physiology 191, 95-104, doi:10.1002/jcp.10080 (2002).

42. Zhang, L., Chen, J. \& Fu, H. Suppression of apoptosis signal-regulating kinase 1-induced cell death by 14-3-3 proteins. Proceedings of the National Academy of Sciences of the United States of America 96, 8511-8515 (1999).

43. Goldman, E. H., Chen, L. \& Fu, H. Activation of apoptosis signal-regulating kinase 1 by reactive oxygen species through dephosphorylation at serine 967 and 14-3-3 dissociation. The Journal of biological chemistry 279, 10442-10449, doi:10.1074/jbc. M311129200 (2004).

44. Subramanian, R. R. et al. Interaction of apoptosis signal-regulating kinase 1 with isoforms of 14-3-3 proteins. Experimental cell research 294, 581-591, doi:10.1016/j.yexcr.2003.12.009 (2004).

45. Schlaepfer, D. D. \& Mitra, S. K. Multiple connections link FAK to cell motility and invasion. Current opinion in genetics es development 14, 92-101, doi:10.1016/j.gde.2003.12.002 (2004).

46. Cance, W. G., Kurenova, E., Marlowe, T. \& Golubovskaya, V. Disrupting the scaffold to improve focal adhesion kinase-targeted cancer therapeutics. Science signaling 6, pe10, doi:10.1126/scisignal.2004021 (2013).

47. Parsons, J. T. Focal adhesion kinase: the first ten years. Journal of cell science 116, 1409-1416 (2003).

48. Hinds, T. D. Jr. \& Sanchez, E. R. Protein phosphatase 5. Int J Biochem Cell Biol 40, 2358-2362, doi:10.1016/j.biocel.2007.08.010 (2008).

49. von Kriegsheim, A., Pitt, A., Grindlay, G. J., Kolch, W. \& Dhillon, A. S. Regulation of the Raf-MEK-ERK pathway by protein phosphatase 5. Nat Cell Biol 8, 1011-1016, doi:10.1038/ncb1465 (2006).

50. Kutuzov, M. A., Andreeva, A. V. \& Voyno-Yasenetskaya, T. A. Regulation of apoptosis signal-regulating kinase 1 (ASK1) by polyamine levels via protein phosphatase 5. J Biol Chem 280, 25388-25395, doi: M413202200 (2005)

51. Liu, Q., Wilkins, B. J., Lee, Y. J., Ichijo, H. \& Molkentin, J. D. Direct interaction and reciprocal regulation between ASK1 and calcineurin-NFAT control cardiomyocyte death and growth. Molecular and cellular biology 26, 3785-3797, doi:10.1128/ MCB.26.10.3785-3797.2006 (2006).

52. Zhao, J. et al. SCF E3 ligase F-box protein complex SCF(FBXL19) regulates cell migration by mediating Racl ubiquitination and degradation. FASEB J 27, 2611-2619, doi:10.1096/fi.12-223099 (2013).

53. Li, L., Lu, Y., Stemmer, P. M. \& Chen, F. Filamin A phosphorylation by Akt promotes cell migration in response to arsenic. Oncotarget 6, 12009-12019, doi:10.18632/oncotarget.3617 (2015).

54. Spurzem, J. R. et al. Activation of protein kinase A accelerates bovine bronchial epithelial cell migration. American journal of physiology. Lung cellular and molecular physiology 282, L1108-1116 (2002).

55. Wyatt, T. A., Ito, H., Veys, T. J. \& Spurzem, J. R. Stimulation of protein kinase C activity by tumor necrosis factor-alpha in bovine bronchial epithelial cells. Am J Physiol 273, L1007-1012 (1997).

56. Slager, R. E. et al. Hog barn dust slows airway epithelial cell migration in vitro through a PKCalpha-dependent mechanism. American journal of physiology. Lung cellular and molecular physiology 293, L1469-1474, doi:10.1152/ajplung.00274.2007 (2007).

57. Desai, L. P., Chapman, K. E. \& Waters, C. M. Mechanical stretch decreases migration of alveolar epithelial cells through mechanisms involving Rac1 and Tiam1. American journal of physiology. Lung cellular and molecular physiology 295, L958-965 (2008).

58. Dobbs, L. G. Isolation and culture of alveolar type II cells. Am J Physiol 258, L134-147 (1990).

59. Ikeda, K. et al. Immortalization of subpopulations of respiratory epithelial cells from transgenic mice bearing SV40 large $\mathrm{T}$ antigen. Am J Physiol 267, L309-317 (1994).

60. Messier, E. M., Mason, R. J. \& Kosmider, B. Efficient and rapid isolation and purification of mouse alveolar type II epithelial cells. Experimental lung research 38, 363-373, doi:10.3109/01902148.2012.713077 (2012).

61. Desai, L. P., Aryal, A. M., Ceacareanu, B., Hassid, A. \& Waters, C. M. RhoA and Racl are both required for efficient wound closure of airway epithelial cells. American journal of physiology. Lung cellular and molecular physiology 287, L1134-1144 (2004).

62. Bradford, M. M. A rapid and sensitive method for the quantitation of microgram quantities of protein utilizing the principle of protein-dye binding. Anal Biochem 72, 248-254, doi: S0003269776699996 (1976).

\section{Acknowledgements}

These studies were supported by National Heart, Lung, and Blood Institute grant HL-123540 (CMW).

\section{Author Contributions}

M.C.G. and C.M.W. conceived and designed the research; M.C.G., P.S.M., J.K., B.T., and C.L. performed experiments; M.C.G. and C.M.W. analyzed the data; M.C.G., C.M.W., P.S.M., and S.E.S. interpreted the results of the experiments; M.C.G. and C.M.W. prepared the figures; M.C.G. and C.M.W. drafted the manuscript; M.C.G. and C.M.W. approved the final version of the manuscript.

\section{Additional Information}

Supplementary information accompanies this paper at doi:10.1038/s41598-017-02204-2

Competing Interests: The authors declare that they have no competing interests.

Publisher's note: Springer Nature remains neutral with regard to jurisdictional claims in published maps and institutional affiliations. 
(i) Open Access This article is licensed under a Creative Commons Attribution 4.0 International License, which permits use, sharing, adaptation, distribution and reproduction in any medium or format, as long as you give appropriate credit to the original author(s) and the source, provide a link to the Creative Commons license, and indicate if changes were made. The images or other third party material in this article are included in the article's Creative Commons license, unless indicated otherwise in a credit line to the material. If material is not included in the article's Creative Commons license and your intended use is not permitted by statutory regulation or exceeds the permitted use, you will need to obtain permission directly from the copyright holder. To view a copy of this license, visit http://creativecommons.org/licenses/by/4.0/.

(C) The Author(s) 2017 